Western University

Scholarship@Western

Psychology Publications

Psychology Department

$10-2017$

\title{
Taking a Person-Centered Approach to Personality: A Latent-Profile Analysis of the HEXACO Model of Personality
}

\author{
Kabir N. Daljeet \\ Western University \\ Nicholas L. Bremner \\ Western University \\ Erica A. Giammarco \\ Western University \\ John P. Meyer \\ Western University, meyer@uwo.ca \\ Sampo V.Paunonen
}

Follow this and additional works at: https://ir.lib.uwo.ca/psychologypub

Part of the Psychology Commons

Citation of this paper:

Daljeet, K.N., Bremner, N.L., Giammarco, E.A., Meyer, J.P., \& Paunonen, S.V. (2017). Taking a personcentered approach to personality: A latent-profile analysis of the HEXACO model of personality. Journal of Research in Personality, 70, 241-251. https://doi.org/10.1016/j.jrp.2017.08.003. 
Taking a Person-Centered Approach to Personality: A Latent-Profile Analysis of the HEXACO

\section{Model of Personality}

Kabir N. Daljeet ${ }^{\mathrm{a}^{*}}$, Nicholas L. Bremner ${ }^{\mathrm{a}}$, Erica A. Giammarco ${ }^{\mathrm{a}}$, John P. Meyer ${ }^{\mathrm{ab}}$, \& Sampo V. Paunonen ${ }^{\mathrm{a}}$

${ }^{a}$ University of Western Ontario, 1151 Richmond Street, London, Ontario, Canada

${ }^{\mathrm{b}}$ Curtin Business School, Curtin University, Perth, Western Australia, Australia

*Corresponding Author. E-mail address: Kdaljeet@uwo.ca (K.N. Daljeet)

This is the prepublication version of the following manuscript:

Daljeet, K.N., Bremner, N.L., Giammarco, E.A., Meyer, J.P., \& Paunonen, S.V. (2017). Taking a personcentered approach to personality: A latent-profile analysis of the HEXACO model of personality. Journal of Research in Personality, 70, 241-251. Doi.org/10.1016/j.jrp.2017.08.003

(C) 2017. This paper is not the copy of record and may not exactly replicate the authoritative document published in the Journal of Research in Personality. 


\begin{abstract}
Our study applies a person-centered approach to the HEXACO model of personality using latent profile analysis (LPA). While the traditional variable-centered approach assumes that the relations among variables within a population are homogenous, the person-centered approach identifies subgroups within samples that have similar scores on several variables of interest, in this case, the six factors of personality. Data from two independent samples were collected at a large North American university. The results of LPA revealed five distinct and interpretable profiles that replicated and were found to be consistent across both samples. We discuss how our findings attest to the meaningfulness of personality profiles, and suggest additional ways in which a person-centered approach might be applied in personality research.
\end{abstract}

Key Words: Person-Centred; Personality; Profiles; Latent Profile Analysis; HEXACO;

\title{
1. Introduction
}

Over the last two decades an increasing number of researchers have begun to take a personcentered approach to the study of personality (e.g., Asendorpf, Borkenau, Ostendorf, \& van Aken, 2001; Herzberg \& Roth, 2006; Isler, Liu, Sibley, \& Fletcher, 2016). The person-centered approach differs from, and complements, the more traditional-variable centered approach in several respects. A primary objective in the variable-centered research is to use personality traits (or trait dimensions), individually or in combination, to predict and explain other variables of interest (e.g., attitude, emotions, and behavior). An underlying assumption in this approach is that the relations observed among personality traits, and between these traits and other variables, are uniform within a given population. In contrast, the personcentered approach operates under the assumption that there may be multiple unobserved subgroups within a population, and that trait relations may differ across subgroups. The objective in this approach, therefore, is to identify these subgroups based on their distinct configurations of trait scores. Importantly, this raises the possibility that a trait might be expressed differently, and relate differently to other variables, depending on the strength of other traits within a profile.

Most of the person-centered personality research to date has been conducted using the Five Factor Model (FFM) of personality (e.g., Asendorf et al., 2001; Merz \& Roesch, 2011; Roth \& von Collani, 2007; Specht, Luhmann, \& Geiser, 2014). This research has purportedly been quite consistent in identifying three distinct configurations of Conscientiousness, Extraversion, Openness, Agreeableness and Emotional Stability (or its inverse, Neuroticism). The three profiles have typically been labeled Resilient, Overcontrolled, and Undercontrolled, based on Block's (1971; Block \& Block, 1980) theory of self-regulation. More recently, however, some investigators have reported finding a larger set of profiles, and have raised questions about whether the three-profile structure is as consistent across studies as initially believed (e.g., Conte, Heffner, Roesch, \& Aasen, 2017; Herzberg \& Roth, 2006; Roth \& von Collani, 2007).

Although the FFM continues to be the most widely accepted taxonomy of personality traits, an alternative six-factor model has more recently been introduced. The HEXACO (Ashton \& Lee, 2005, 2008; Lee \& Ashton, 2004) encompasses higher-order traits like the FFM, but uses slightly different terminology (e.g., Emotionality versus Neuroticism). Most importantly, the HEXACO expands the personality space by including a sixth dimension, Honesty-Humility. Honesty-humility has been linked to a number of important variables such as job performance, counterproductive work behavior, well-being, leadership, organizational citizenship behaviors, and social dominance orientation (Aghababaei \& Arji, 2014;Bourdage, Lee, Lee, \& Shin, 2012; De Vries, 2012; Johnson, Rowatt, Petrini, 2011; Sibley, Harding, Perry, Asbrock, \& Duckitt, 2010) and has been found to explain additional variance over and above the traits of the FFM (De Vries, De Vries, De Hoogh, \& Feij, 2009; Lee, Ashton, \& De Vries, 2005; Marcus, Ashton, \& Lee, 2013; Marcus, Lee \& Ashton, 2007).

We are aware of only two person-centered studies to date conducted using the HEXACO dimensions (Aston \& Lee, 2009; Isler et al., 2016), and the investigators came to quite different conclusions about the nature and meaningfulness of the underlying profile structure. Consistency is 
arguably an important criterion in evaluating the meaningfulness of the profiles identified in personcentered research (Herzberg \& Roth, 2006; Morin, Meyer, Creusier, \& Biétry, 2016), and there are too few HEXACO studies at this point to draw any firm conclusions. Therefore, our objective in this study was to investigate the profile structure of the HEXACO using two comparable samples and to examine cross-sample consistency using a set of procedures recently introduced by Morin et al. (2016). We elaborate on these procedures, and the person-centered approach in general, below. We then provide a summary and critique of previous person-centered studies involving the FFM. This is followed by a brief discussion of the differences between the FFM and the HEXACO, a summary of the extant HEXACO studies, and a statement of our study hypotheses.

\subsection{The Person-Centered Approach}

As noted previously, the person-centered research strategy is built on the assumption that a defined population of people (and samples drawn from it) might include multiple unobserved subgroups with distinct configurations of variables (e.g., traits). Identifying these subgroups and understanding the nature and implications of the trait configurations that distinguish them can be an important complement to the variable-centered strategies that have been dominant to date (Wang \& Hanges, 2011; Zyphur, 2009). Perhaps the biggest advantage of the person-centered approach is that it helps to address potentially complex relations among personality traits, and/or between these traits and other variables. For example, a variable-centered approach can inform us that, on average, trait $\mathrm{X}$ correlates positively with trait $\mathrm{Y}$ within a population. However, a person-centered approach can identify subgroups within that population where traits $\mathrm{X}$ and $\mathrm{Y}$ co-occur, as well as subgroups where they do not. These traits might be expressed quite differently in the two instances. If this is the case, then the results of variable-centered research examining relations between individual trait dimensions and other variables of interest (e.g., academic or job performance) could be misleading. Although it is possible to address some of these complexities by examining interactions among traits in variable-centered studies, these become difficult to detect and interpret as interactions become more complex (McClelland \& Judd, 1993). These complex interactions are more easily captured and conveyed by identifying subgroups using a person-centered approach and comparing these groups on other variables of interest (Asendorpf, 2015; Meyer \& Morin, 2016).

There are various ways that person-centered analyses can be conducted, but the most common include cluster analyses and latent profile analyses (LPA). Detailed comparison of these analyses go beyond the scope of the present article (see Morin, Morizot, Boudrias, \& Madore, 2011, and Pastor, Barron, Miller, \& Davis, 2007, for more detail), but it is generally recognized that LPA is superior in many respects. It is a model-based technique and various statistical indicators are available to assist in selecting the optimal model from among various alternatives. Moreover, individuals are assigned values (posterior probabilities) reflecting the likelihood of membership in each profile group, and these values can be used in subsequent analyses in making group comparisons (for a more detailed discussion of LPA and a guide to its use, see Meyer $\&$ Morin, 2016). There are also procedures available to compare profiles structures across samples (Morin et al., 2016). For these reasons, we used LPA in the present study.

It is important to acknowledge that LPA is essentially exploratory, at least from an analytic perspective (Meyer \& Morin, 2016; Wang \& Hanges, 2011). That is, there are no conventional goodness of fit statistics such as the Comparative Fit Index (CFI) or Root Mean Squared Error of Approximation (RMSEA) available to evaluate the absolute goodness of fit. Therefore, it is standard practice to extract and compare multiple solutions using the various statistical indicators mentioned above. Evaluation of the substantive meaning of the profiles can also serve as a guide. Such an evaluation can be based on theory when it exists, and/or demonstration that the same profiles emerge consistently across samples and/or relate in meaningful ways to other variables of interest. As we discuss in more detail below, extraction and interpretation of profiles in previous person-centered studies of the FFM and HEXACO have been guided by theory, but there are reasons to question whether this is fully appropriate. In the present study, we rely on evidence of profile consistency in evaluating substantive meaning.

Profile consistency can take different forms, including consistency across populations, across samples within populations, and across time. Of these, consistency across samples within populations is 
arguably most important from a meaningfulness perspective. In the case of personality, it is possible that the nature of the heterogeneity will vary across populations differing in culture, age, socioeconomic status, and the like. Thus, finding different profile groups across distinct populations does not undermine the meaningfulness of profiles within a population. It is also possible to identify different, yet meaningful, profiles within a sample over time, particularly if the interval is long and/or it encompasses a particularly crucial period of development (e.g., the transition for adolescence to adulthood) or an intervention (e.g., therapy). By contrast, failure to find consistency in the profile structure across samples drawn from the same, or a very similar, population would raise some question about whether configurations detected are spurious. In the present study, we compared the profile structure for two samples of students drawn from essentially the same population in two consecutive academic years. To compare the profile structures, we applied procedures recently developed by Morin et al. (2016). Details regarding the steps involved in this comparison process are presented in the Analysis section below.

\subsection{Person-Centered Studies of the FFM Model}

There have been several studies conducted to identify personality profiles based on the FFM dimensions, and these have generally yielded between three and five profiles (e.g., Asendorf et al., 2001; Merz \& Roesch, 2011; Roth \& von Collani, 2007; Specht et al., 2014). Interpretation of these profiles has been guided largely by Block's (1971; Block \& Block, 1980) self-regulation theory. Indeed, in several of the early studies (e.g., Gramzow et al., 2004; Robins, John, Caspi, Moffitt, \& Stouthamer-Loeber, 1996) person-centered analyses (e.g., inverse factor analyses) were conducted using a Q-Sort measure scored in terms of Ego-resilience and Ego-control. Ego-control is a bi-polar construct and reflects the tendency to freely express (undercontrol), or inhibit (overcontrol), the expression of one's motives and emotions. Egoresilience reflects the degree of elasticity of one's self-regulatory system, or the ability to modulate one's natural control tendencies (free expression or inhibition) based on situational demand. Once profile groups were identified from the Q-Sort data, they were compared on FFM dimension means.

In these early studies, the profile labels were derived from the Q-Sort profiles using terminology drawn from Block's (1971) self-regulation theory. These same labels were then applied to the corresponding configurations (profiles) of the FFM dimensions identified for each group. For example, Robins et al. (1996) identified three Q-Sort profiles and labeled these resilient, overcontrolled, and undercontrolled, respectively. They found that individuals with the resilient profile had relatively high scores on all five of the FFM traits, those with the overcontrolled profile had relatively low scores on extraversion and emotional stability, and a relatively high score on agreeableness, and those with an undercontrolled profile had relatively high scores on extraversion and low scores on agreeableness and conscientiousness. Gramzow et al. (2004) found four Q-Sort profiles and labeled them resilient undercontrollers, resilient overcontrollers, brittle undercontrollers and brittle overcontrollers. When compared on the FFM traits, those with more resilient profiles scored low on neuroticism (high on emotional stability) and those with brittle profiles scored high on neuroticism. Those with overcontrolled profiles scored relatively low on extraversion and openness, whereas those with undercontrolled profiles scored high on extraversion and on either openness or agreeableness.

Subsequent studies applying person-centered analyses (typically cluster analyses or LPA) to the FFM traits directly have applied labels like those found in the Q-Sort studies. Indeed, the 'resilient,' 'overcontrolled,' and 'undercontrolled' labels have been used quite consistently in studies that identified three profiles (e.g., Asendorpf et al., 2001; Barbaranelli, 2002: Chapman \& Goldenberg, 2011; Specht et al. 2014). Studies identifying more than three profiles typically use some variation of these three labels (e.g., Conte et al., 2017; Herzberg \& Roth, 2006; Roth \& von Collani, 2007). However, close inspection of the profiles obtained in these studies reveals considerable variability in the actual patterns of FFM dimension means, thereby raising questions about the appropriateness of a labeling scheme based on Block's (1971) self-regulation theory. For example, Specht et al. (2014) used the 'overcontrolled' label for a profile with low emotional stability, extraversion and openness scores in German samples of men and women, for a profile with low emotional stability and high openness scores in a sample of Australian men, and for a profile with no extreme scores in a sample of Australian women. In other cases, the same labels are used across samples regardless of whether it was conscientiousness, openness, or agreeableness, 
or some combination of these, that defined the profile (e.g., Asendorpf et al., 2001). Gramzow et al. (2004, p. 369) noted that the FFM evolved as a way "to identify the key dimensions along which individual differences in emotion, thought, and behavior occur." Consequently, although variation along these dimensions might be expected to reflect the self-regulatory processes described by Block (1971) to some extent, there are also likely to be other contributing factors. It is therefore not surprising that the profiles obtained directly from analyses of the FFM do not always correspond to what might be expected based on self-regulation theory.

The fact that studies with the FFM traits differ in the number and nature of profiles identified might raise concerns about their meaningfulness. However, it should be noted that the samples included in these studies differ considerably in terms of size, age, gender composition, and national culture. The studies also differ in terms of the measures used and the analytic strategies applied. As we noted above, there may be true differences across populations in the extent to which specific profiles are represented. Consequently, it might be impossible to identify all potentially meaningful trait configurations within a single study (cf. Herzberg \& Roth, 2007). What is particularly important, therefore, is to demonstrate that there is consistency in the profiles across samples drawn from the same population. There is some evidence from the FFM studies to suggest that is indeed the case. For example, several investigators demonstrated consistency using a split-sample technique (Assendorpf et al., 2001; Barbaranelli, 2002; Herzberg \& Roth, 2006).

\subsection{Person-centered Studies of the HEXACO Model}

As noted previously, the HEXACO model (Lee \& Ashton, 2004) encompasses higher-order traits like those in the FFM. The most obvious difference between the FFM and the HEXACO is the inclusion of an Honesty-Humility dimension in the latter. Individuals with high scores on Honesty-Humility tend to be honest, sincere, fair, and modest, whereas those with low scores are greedy, conceited, deceitful, and pretentious. Inclusion of the Honesty-Humility dimension was accompanied by adjustments in the conceptualization of Agreeableness and Neuroticism (described in the HEXACO as Emotionality). Individuals with high scores on Agreeableness tend to be peaceful, gentle, patient and agreeable, whereas those with low scores are quick-tempered, choleric, stubborn, and quarrelsome. Although it resembles the FFM dimension, Agreeableness in the HEXACO model lacks the sentimentality component at the positive pole, and the anger component at the negative pole, of the FFM version. Individuals with high scores on Emotionality tend to be vulnerable, sensitive, anxious and sentimental, whereas those with low score are fearless, tough, independent and unemotional. Thus, this dimension is quite different from the Neuroticism (versus Emotional Stability) dimension in the FFM and must be considered accordingly when interpreting HEXACO profiles. The remaining three dimensions, Extraversion, Conscientiousness and Openness are quite similar in the FFM and HEXACO models.

To date, there have been only two person-centered studies of the HEXACO dimensions, and the results have been mixed. The first study was conducted by Ashton and Lee (2009) using cluster analysis. They explored several different cluster solutions, and compared the variance in the personality dimensions explained by these clusters with that explained by clusters observed from randomly-generated data. They concluded that that there "is no clear clustering of individuals within the space of the six HEXACO-PI dimensions" (p. 185).

More recently, Isler et al. (2016) applied LPA to data obtained using the mini IPIP, a 20-item measure of the FFM dimensions from the International Personality Item Pool (IPIP), and 4 additional items to measure Honest-Humility and approximate the HEXACO. The measures were administered on two separate occasions to a randomly selected sample of registered voters in New Zealand. As in most of the earlier studies involving the FFM, Isler et al. proposed that the profiles obtained with the HEXACO would reflect the joint operation of Ego-control and Ego-resilience. However, they proposed, and found, that a 4-profile solution provided a better fit to the data than the 3-profile solution commonly found in the FFM studies (i.e., resilient, overcontrolled, undercontrolled). They used the label 'brittle' to describe the fourth profile where scores on most dimensions were in the undesirable direction.

Isler et al. (2016) explained the superiority of the 4-profile solution in terms of its ability to more accurately capture the true interaction between Ego-control and Ego-resilience. Little attention was paid 
to the potential influence of the inclusion of the Honesty-Humility dimension. However, on closer inspection, it is not clear that the labeling system used by Isler et al. is appropriate. For example, they used the label 'overcontrolled' to describe a profile defined by low Extraversion and high HonestyHumility. Similarly, they used the label 'undercontrolled' to describe a profile characterized by low Honesty-Humility alone. These profiles were not defined by high and low scores on Openness or Agreeableness as they were described in the early Q-Sort studies (e.g., Gramzow et al., 2004; Robins et al., 1996). Given the nature of the Ego-control construct (Block, 1971) described above, it is not clear that Honesty-Humility is a good substitute for Openness and/or Agreeableness as a defining characteristic.

Regardless of the labeling issue, it is important to note that Isler et al. (2016) also demonstrated consistency in the profile structure within the sample over time. Moreover, using latent transition analysis (LTA), they demonstrated a high degree of within-person consistency. Less than one percent of the sample changed profile membership over time. As noted previously, evidence for such consistency helps to bolster the argument that the observed profiles reflect meaningful rather than spurious configurations among the trait dimensions. However, given the paucity of attention given to date to HEXACO profiles, additional research is warranted.

\subsection{The Present Study}

The fact that, as with the FFM studies, Isler et al (2016) could identify multiple profiles using the HEXACO, and that these remained relatively stable over time, suggests that there might indeed be subgroups with meaningfully different configurations (profiles). Our objective in the present research was to use LPA to identify HEXACO profiles in a different population for comparison with those identified by Isler et al. Although finding similar profiles in a different population would provide particularly strong evidence that there is indeed a meaningful profile structure underlying the HEXACO, this is not a requirement. For reasons stated above, the profile structure might differ across distinct populations. Our methodology and analytic techniques also differed in several respects (e.g., measures, use of latent variables). For these reasons, we did not hypothesize that we would replicate the structure identified by Isler et al. From a meaningfulness standpoint, it is more important to demonstrate that the profile structure is consistent across samples drawn from the same population. To this end, we conducted LPA with two such samples and used the analytic techniques developed by Morin et al. (2016) to evaluate profile consistency. Thus, our objective was to test the following hypotheses:

Hypothesis 1: The application of LPA to HEXACO data will identify multiple profiles with different configurations of the six dimensions.

Hypothesis 2: The same number of profiles with the same configurations of dimensions will be obtained in two samples drawn from the same population.

\section{Methods}

\subsection{Participants}

Participants in this study were undergraduate psychology students who signed up to complete a large test battery in exchange for course credit. Participants were recruited in two waves (one year apart) from an online research participation pool. In total, 2,633 participants were recruited for this study; however, after removing careless responders (see below for more details), 1,738 participants remained, 880 from the first sample and 858 from the second sample. In addition to demographics, participants completed the measure of personality described below. Samples 1 and 2 were similar in terms of gender composition (63.10\% and 62.70\% female, respectively), age (sample 1: $M=18.28, S D=1.53$; sample 2: $M=18.32, S D=1.63)$, and percentage of employed participants (19.00\% and $18.20 \%$, respectively).

\subsection{Materials}

2.2.1 Demographics. Participants provided information regarding their age, gender, and employment status.

2.2.2 Personality. Participants completed the 60-item self-report HEXACO personality inventory (Ashton \& Lee, 2009). This measure contains six, 10-item subscales: Honesty-Humility, Emotionality, Extraversion, Agreeableness, Conscientiousness, and Openness. Participants responded using a 5-point Likert-type scale ranging from 1 (Strongly Disagree) to 5 (Strongly Agree). Ashton and Lee (2009) found 
the internal consistency reliability for each of the subscales to be above .73 and also provided evidence in support of the measure's validity.

2.2.3 Careless Responding. In accordance with suggestions made by Meade and Craig (2012), participants in both samples were asked to respond to three items directing them to make a particular response (e.g., "Please respond Strongly Agree to this item"). These items were distributed throughout the test battery and participants who failed to respond to one or more of these items with the correct response were excluded from analyses.

\subsection{Analyses}

The analyses were conducted in three stages. First, following procedures recommended by Vandenberg \& Lance (2000), we conducted a multi-group confirmatory factor analysis (CFA) on the HEXACO measures for the two samples. This analysis served two purposes. First it allowed us to evaluate measurement invariance across the samples. Second, it provided a set of factor scores to use as input for the LPA.

Testing for measurement equivalence is a sequential process involving the evaluation of four nested forms of invariance: configural, metric, scalar, and error. Configural invariance is the most basic form and is established when the number of factors in the optimal CFA solution is the same for both samples. Metric invariance is presumed to exist when the loadings of the indicators (personality items) on each factor are similar across groups. Scalar invariance is established when the latent variable intercepts are found to be equivalent, and error invariance is established when the measurement error parameters are found to be equivalent. Progressing to the next step in the sequence requires evidence of equivalence at the previous level. Testing for equivalence in each of the last three steps involves comparison of the fit of two models, one in which the relevant parameters are fixed to be equal across samples and the other in which the parameters are freely estimated (i.e., as in the model supported in the previous step). If the fit of the two models is not significantly different, equivalence is presumed to exist. For purposes of subsequent analyses, we required evidence of configural and metric equivalence at a minimum.

The second stage of analysis involved conducting LPA separately for the two samples. For this analysis, we used factor scores for the model with the highest degree of measurement invariance established in the multi-group CFA. Using latent factor scores helps to partially control for measurement error and improves the generalizability of the LPA across groups (Morin et al., 2016). LPA was conducted using Mplus 7 (Muthén \& Muthén, 1998-2012) using robust maximum-likelihood (MLR) estimation. LPA identifies groups of cases (or individuals) in a sample possessing similar values on a given set of variables (Muthén, 2001). Each profile is represented as a latent categorical variable that explains the correlations among its indicator variables, which in this case correspond to the six subscales of the HEXACO personality inventory. LPA provides fit statistics to assist in determining the optimal number of profiles for any given sample. This is done via an iterative process where models with increasing numbers of profiles are specified until the profile solutions and fit statistics become less favorable. To evaluate the fit of each model, we examined the Akaike Information Criterion (AIC; Akaike, 1987), the Consistent AIC (CAIC; Bozdogman, 1987), the Bayesian Information Criterion (BIC; Schwartz, 1978), and the sample-size adjusted BIC (SABIC; Sclove, 1987). Lower values for these statistics represent a superior fit. We also examined the Lo, Mendell, and Rubin (2001) likelihood ratio test (LMR), and the Bootstrap Likelihood Ratio Test (BLRT; McLachlan \& Peel, 2000) for each solution. For both of these tests, a significant $p$ value indicates that a $k$ profile solution is superior to a solution containing the $k-1$ profiles (McLachlan \& Peel, 2000). Finally, we evaluated the Entropy and posterior probabilities for each solution to determine how accurately cases were assigned to their respective profiles (Nylund, Asparouhov, \& Muthén, 2007). Although Entropy should not be used to evaluate the suitability of a profile solution (Lubke \& Muthén, 2007), it was included as a useful summary of classification accuracy. Specifically, Entropy indicates the precision with which the cases are classified into profiles, with higher values (from 0 to 1 ) indicating fewer classification errors. Nylund et al. (2007) recommend that the optimal profile solution should be determined not only by examining fit statistics, but also by examining profile size, and distinctiveness. This is because solutions with a larger number of profiles will often continue to produce slightly superior fit statistics, but the configuration (or appearance) of profiles 
will often begin to look quite similar to one another. Accordingly, all of these factors were taken into account when choosing our final profile solution for each sample. The LPA for each sample provided the test of Hypothesis 1.

In the final stage, we conducted a multi-group LPA to evaluate the profile similarity across the two samples. As in the test of measurement invariance, testing for profile similarity is a sequential process. Morin et al. (2016) recently identified six forms of similarity and described the procedures use to evaluate them. Only the first four are relevant in the present study; the later forms involve comparisons of parameters for potential predictors and outcomes of profile membership. Configural similarity is established when it can be shown that the optimal solution for two samples includes the same number of profiles. Structural similarity exists when the means of each indicator within profiles are the same across samples. Similarly, dispersion similarity exists when the variances for each indicator within profiles are the same across samples. Finally, distributional similarity is established when the proportions of individuals within each profile is equivalent across samples. Each of the last three tests involves the comparison of a model in which the relevant parameters are constrained with the unconstrained model from the previous step. The relative fit of these models is used to determine whether the constraints are supported and similarity is indicated. Support for Hypothesis 2 requires that, at a minimum, configural and structural similarity have been established.

\section{Results}

Means, standard deviations, reliabilities, and correlations among the study and demographic variables are presented in Table 1.

\subsection{Multi-group CFA}

Multi-group CFA was used to simultaneously establish and test the baseline (6-factor) model in both samples. The model demonstrated adequate fit $\left(\chi^{2}[3390, \mathrm{~N}=1738]=11506.15, p<.001, \chi^{2} / \mathrm{df}=\right.$ $3.39, \mathrm{CFI}=.64, \mathrm{RMSEA}=.05)$, thereby establishing configural invariance. The model was then further constrained to test for metric invariance. The metric invariant model fit the data well $\left(\chi^{2}[3444, \mathrm{~N}=1738]\right.$ $\left.=11543.32, p<.001, \chi^{2} / \mathrm{df}=3.35, \mathrm{CFI}=.64, \mathrm{RMSEA}=.05\right)$, thereby establishing metric invariance. We were unable to establish stricter forms of measurement invariance by testing further constrained models. Accordingly, we saved factor scores produced by the metric-invariant model as input for the LPA.

\subsection{Latent Profile Analysis}

The fit statistics for the 2- to 8-profile solutions for each of the two samples are reported in Table 2. The percentage of the sample assigned to each profile are reported in Table 3 , and posterior probabilities reflecting the accuracy of classification to each profile are presented in Table 4 . As illustrated in Table 2, AIC, CAIC, BIC, and SABIC values steadily decreased for both samples up to the 5-profile solution. For Sample 1, the BIC began increasing after the 5-profile solution and the remaining fit statistics continued to decrease, but at a much slower rate, which points to the superiority in fit for the 5-profile solution. For Sample 2, all fit statistics continued to decrease after the 5-profile solution, but at a slower rate, suggesting that improvements in model fit diminished after this point. The significance levels of the LMR were not quite as straightforward to interpret given that estimates went from being significant to nonsignificant and then back to significant again (a common occurrence according to Nylund et al., 2007). The last significant $p$ value for the LMR points to the 4-profile solution as the best fit for Sample 1, and to the 6-profile solution as the best fit for Sample 2. For both samples, the BLRT was not particularly helpful in determining the best fitting profile solution as the value remained significant up to the 8-profile solution, suggesting that even more profiles would produce a better fit. However, Marsh, Lüdtke, Trautwein, and Morin (2009) emphasized that, when sample sizes are large, the BLRT will often favor a larger number of profiles, even if the additional profiles extracted are highly similar. Further support for the 5-profile solution was evidenced by the fact that the 6- and 7-profile solutions extracted profiles consisting of less than $5 \%$ of the total sample (in both samples; see Table 3), which falls below the cutoff recommended by Nylund et al. (2007). The posterior probabilities for the 5-profile solutions in both samples indicated that there is a high likelihood that participants were accurately assigned to the correct profiles (see Table 4). Finally, we examined the distinctiveness of the profiles from the 4-, 5-, 6-, and 7-profile solutions. For the 5-profile solution, all profiles were distinct in terms of shape (i.e., the 
pattern of high and low mean scores of each personality trait differ). When six or more profiles were extracted, some of the profiles reflect only very small differences in shape (i.e., the relative pattern of mean scores are the same, but differ slightly in elevation). Thus, all things considered we determined that the preponderance of evidence indicates that the 5-profile solution was optimal for both samples, thereby supporting Hypothesis 1. The next step in the analysis, therefore, was to determine whether the same 5profile solution could be fit to the two samples. ${ }^{1}$

\subsection{Profile Similarity}

The test of configural similarity was supported given that the 5-profile solution was judged to be optimal for both samples. The fit statistics for the configural invariance model and remaining models testing the other three forms of invariance (structural, dispersion, and distributive) are presented in Table 5 .

As compared to the configural similarity model, the structural similarity model had lower AIC, BIC, SABIC, and CAIC values, and was therefore a superior fit. This supported the structural similarity of the two profile solutions. Next, the dispersion similarity model was run and demonstrated a mixed improvement in fit over the structural similarity model. While the AIC statistic was slightly higher, the dispersion similarity model had lower BIC, SABIC, and CAIC values, thereby supporting dispersion similarity between the two samples. Finally, the model of distributional similarity demonstrated further improved fit statistics across the board as compared to the dispersion model. Accordingly, given that the best-fitting profile solutions for both samples were found to be equivalent in terms of profile number, as well as within-profile means, variability, and size, Hypothesis 2 was supported.

\subsection{Profile Labels}

We used the pattern of dimension means from the 5-profile dispersion invariant model in the multi-sample comparison for purpose of labeling the profiles. These means are depicted graphically in Figure 1.

When deciding on labels, we carefully examined the definitions of the HEXACO dimensions and the trait facets associated with each core trait (Lee \& Ashton, 2004, 2014, 2016). The first profile was characterized by above average Honesty-Humility, Agreeableness, Conscientiousness and Emotionality. Honesty-Humility and Agreeableness have been reported to share variance and reflect a pro-social orientation. In this context, Conscientiousness might also be interpreted as reflecting a pro-social orientation and therefore we labeled this profile Socially Considerate. Profile 2 was defined primarily by above average scores on Extraversion and Agreeableness, and below average scores on Emotionality, Honesty-Humility, and Conscientiousness. This pattern reflects an outgoing personality and perhaps a greater proclivity for social interaction than for social responsibility. We labeled this profile SelfConfident.

Profile 3 is characterized most clearly by above average scores on Emotionality and Conscientiousness, and below average scores on Agreeableness and Honesty-Humility. Individuals with this profile are conscientious but, unlike those in Profile 1, appear to be less socially responsible. In this context, the conscientiousness might have more of a task focus (e.g., on performing well in one's studies). The emotionality in this case might also reflect anxiety surrounding performance. Therefore, we labeled this profile Goal-oriented. Profile 4 is defined by particularly low scores on Conscientiousness and Extraversion, with the remaining scores about average. Individuals with this profile appear to be internally focused (introverted) and, in this context, the low conscientiousness score might reflect a lack of social responsibility. We labeled this profile Withdrawn.

Finally, Profile 5 is defined by above average scores on Extraversion and below average scores on all other dimensions, most notably Honesty-Humility, Agreeableness, and Conscientiousness. Individuals with this personality are outgoing like those in Profile 2, but are decidedly anti-social (in contrast to the pro-social nature of individuals in Profile 1). Individuals with this profile might be characterized by one or more of the Dark Triad traits (Narcissism, Machiavellianism, Psychopathy;

\footnotetext{
${ }^{1}$ The 5-profile solutions for both samples are available from the authors upon request
} 
Paulhus \& Williams, 2002). However, in the absence of more specific data, we labeled this profile Maladjusted.

\section{Discussion}

Person-centered studies of the FFM dimensions consistently reveal between three and five subgroups with distinct trait configurations, or profiles. Although the number and nature of the profiles differs somewhat across studies, this might be attributable to differences in the size and nature (e.g., culture, age- and gender-composition) of the samples. When profiles were compared across split samples within studies, the findings were generally consistent (e.g., Assendorpf et al., 2001; Barbaranelli, 2002; Herzberg \& Roth, 2006), suggesting that they are not spurious. Very few person-centered studies have been conducted to date using the HEXACO framework, but in the one study using LPA to identify profiles, Isler et al. (2016) found that a similar 4-profile solution fit the data well for the same sample on two separate occasions. Our objective in this study was to investigate further the profile structure of the HEXACO dimensions, and to use recently introduced statistical procedures to compare profiles across samples (Morin et al., 2016).

\subsection{HEXACO Profiles}

Our analyses yielded a 5-profile solution that was consistent across two student samples. Comparison of our profiles with those identified by Isler et al. (2016) reveals several differences. The first and most obvious difference is in the number of profiles. Although there are several possible explanations for this difference, including the use of very different samples (i.e., university undergraduates versus registered New Zealand voters), it is noteworthy that the fit statistics reported by Isler et al. supported the extraction of more than four profiles. They chose the 4-profile solution because interpretability was difficult beyond 4-profiles. This difficulty may have been due to their efforts to base their interpretation on the framework previously established with the FFM. As noted previously, we chose instead to base our decision concerning the optimal profile solution on statistical indicators and replication across samples. Other methodological reasons for the differences in findings include the fact that we used a more comprehensive measure of the HEXACO model of personality (i.e., the 60-item HEXACO PI [Ashton \& Lee, 2009] versus an augmented version of the Mini IPPIP [Donnellan, Frederick, Oswald, \& Lucas, 2006]). Moreover, based on recommendations made by Meyer and Morin (2016), we used factor scores rather than raw scores as input to the LPA. Using factor scores helps to preserve the original measurement structure of a model, which, in turn, leads to more accurate detection of profiles.

Looking beyond number, some of the profiles we identified were quite different in shape and dispersion from those reported by Isler et al. (2016). Again, there are several possible reasons for these differences, including those used to explain differences in the number of profiles. Although it is still too early to draw any firm conclusions regarding the differences in profile structure, it is useful as a guide for future research to consider both the similarities and differences. It is also important to consider the labelling scheme used in the two studies. As noted earlier, there are reasons to question the labeling scheme used in the FFM studies, but with the inclusion of the Honesty-Humility factor, this scheme seems even less tenable for the HEXACO model.

Only the first profile in our 5-profile solution is similar in shape to a profile found by Isler et al. (2016). Our profile included above-average scores on Honest-Humility, Agreeableness, Conscientiousness and Emotionality, and is most like the profile that Isler et al. labelled 'Resilient' (high scores on Agreeableness, Honesty-Humility, and Openness). We chose not to use 'Resilient' as a label because, based on its origins in the early FFM studies (Gamzow et al., 2004; Robins et al., 1996), it implies an ability to adapt one's natural regulatory style (e.g., overcontrolling or undercontrolling) to the situation (Block, 1971). We found little justification for making such an inference in our study. We chose instead to use the label 'Socially Considerate' based on the descriptions of the defining dimensions, and the fact that Honesty-Humility and Agreeableness share some common variance reflecting prosocial tendencies (Ashton et al., 2014). In this context, moderately high scores on Conscientiousness might also be interpreted as demonstrating pro-social tendencies. We used a similar strategy in labeling the remaining profiles in our 5-profile solution (see Profile Labeling section above). We argue that this approach is more consistent with the underlying objectives in the development of the FFM and HEXACO 
frameworks, namely identification of the core dimensions underlying trait covariation (Gamzow et al., 2004), and does not presume that such covariation can be explained by any one theory.

Labeling of profiles is important because it can influence the inferences people are likely to make about the kinds of people they describe. These inferences need to be tested in subsequent research, and labels can ultimately be adjusted based the findings that emerge. One strategy might be to reverse the procedures used by Robins et al. (1996) and Gamzow et al. (2004) in the original labeling of the FFM profiles. Recall that these investigators used a Q-Sort method with measures designed to test Block's (1971) self-regulation theory, and then compared the profile groups on the FFM dimensions. In future research, HEXACO (or FFM) profiles could be generated and then compared on measures designed to reflect the processes specified by various theories of personality development, including Block's (1971) self-regulatory theory. Once we have a clearer understanding of why various configurations emerge, we will be in a better position to assign meaningful labels.

\subsection{Profile Consistency}

Despite our inability at this point to articulate the processes involved in the formation of the five profiles that emerged in our study, we believe that these profiles are indeed meaningful for the population under investigation. As hypothesized, we found that the same five profiles emerged across two samples drawn a year apart from essentially the same population. By following the multi-step procedure laid out by Morin et al. (2016), we demonstrated that the profiles found in the two samples were similar in number, profile shape (i.e., mean scores on the dimensions within profiles), variance of the indicators, and relative size. This level of similarity increases our confidence that the profiles we obtained reflect meaningful ways in which the HEXACO dimensions combine within the population of university students from which we drew our samples. Demonstrating such similarity is an important first step and sets the stage for future research to investigate why these specific patterns emerge and how the different configurations relate to important outcomes. We return to discussion of this issue below.

\subsection{Limitations}

As is the case with all studies, our research has limitations that must be considered when interpreting the results. The most obvious is the sample. We cannot claim that the 5-profiles identified here are representative of the whole structure of personality. Rather, we have identified five meaningful profiles that were robust across two comparable Canadian student samples. Accordingly, we believe these profiles represent a meaningful subset of all possible HEXACO profiles. It is quite possible that additional profiles will be discovered with the use of more diverse samples, or samples drawn from very different populations. The purpose of this paper was to illustrate the viability of taking a person-centered approach to studying personality, and it is our hope that future researchers will apply these techniques in their work. Our study also used consistency across samples as a criterion for determining the meaningfulness of the profiles. It will be important in future research to address meaningfulness in other ways, such determining whether profile membership can be predicted, and whether it in turn helps to predict and explain other variables of interest. We elaborate on this point below.

\subsection{Implications and Future Directions}

The HEXACO model of personality has gained increasing research attention over the past decade because it helps to account for variance in personality not explained by the FFM (e.g., Ashton \& Lee, 2007; Jackson, Paunonen, Fraboni, \& Goffin, 1996; Jones \& Paulhus, 2014; Lee \& Ashton, 2005, 2014; Veselka, Schermer, \& Vernon, 2011). As such, we felt it was important to extend application of the person-centered approach beyond the FFM to determine whether the additional construct coverage in the HEXACO might influence profile structure. Our findings confirm that the profile structure is indeed different from that typically reported in FFM studies. It also differs from the profile structure observed in the one other application of LPA to the HEXACO dimensions of which we are aware (Isler et al., 2016). We attribute the latter finding to the fact that Isler et al. use the earlier FFM studies to guide extraction and labeling of HEXACO profiles.

Admittedly, investigation of HEXACO profiles is at a very early stage and there is reason to question whether the profiles observed in the present study will generalize to other populations. This is an empirical question and it is essential that studies similar to ours be conducted with other populations. 
Although we can only speculate at this point, we expect that some of the profiles we identified will emerge quite consistently. For example, the profile we described as Socially Considerate was large and quite similar in some respects to the Resilient profile reported in most FFM studies. Therefore, we expect that it is likley to replicate. Other profiles might replicate to some extent, but differ in size, elevation, or scatter. For example, consistent with Specht et al. (2014), we might expect a greater proportion of individuals with a Socially Adjusted profile in older samples, presumably reflecting a higher level of maturity. Similarly, the profile we labeled as Withdrawn might have more extreme low scores on Extraversion and Conscientiousness in a clinical population, and the profile we labeled Maladjusted might have higher scores on Extraversion and lower scores on Honesty-Humility and Conscientiousness in a criminal population. Still other profiles might be unique to our sample of students, and studies with other populations might identify profiles not found in our study. This combination of common and unique profiles across populations/samples has been observed in other domains (e.g., organizational commitment: see Kabins, Xu, Bergman, Berry, \& Willson, 2016; Meyer \& Morin, 2016). Identifying the full set of meaningful configurations of HEXACO profiles might require analyses with a very large and diverse sample (cf. Herzberg \& Roth, 2006; Kabins et al., 2016). This would then allow for comparison of profiles obtained with samples drawn from more unique populations.

We noted above that cross-sample consistency is only one criterion, albeit an important one, in evaluating the meaningfulness of personality profiles. It is also important to demonstrate that profile membership has implications for our understanding of individual differences in other variables of interest. For example, research following from the present study of undergraduate students might consider whether, and how, students with different profiles perform academically, interact socially, and/or cope with stress. Studies involving other populations might be concerned with whether profiles can be identified and help in the prediction of recidivism rates among juvenile delinquents, incidents of posttraumatic stress disorder among military personnel, or effective leadership among managerial candidates. Note that such research can be useful even if it is detemined that profile structure is not generalizabile across populations. As long as a population of interest is sufficiently large, and there are important criteria to be predicted, identifying a meaningful profile structure can be useful even if it is somewhat unique.

Another potential direction for future person-centered research might be to expand beyond the FFM and HEXACO dimensions. For example, research into maladaptive personality traits has seen an enormous increase with the introduction of the Dark Triad (Paulhus \& Williams, 2002). It would be interesting to investigate whether these more antisocial aspects of personality are similiarly suited to profile analysis, and if so, how those profiles can be used to predict relevant behavioral outcomes. Another potential application would be at the facet level within the FFM, HEXACO, or Dark Triad models. Some personality theorists have been critical of the 'lumping' of traits into broad categories (Ashton, Jackson, Paunonen, Helms, \& Rothstein, 1995; Paunonen, 1998; Paunonen \& Ashton, 2001; Paunonen, Jackson, \& Rothstein, 1999). By applying LPA to the facet traits within the broader categories, it might be possible to identify meaningful subtypes. For example, there might be different forms of Conscientiousness, with some individuals more concerned with responsibility and self-control, and others with achievement and persistence. In addition to addressing theoretical debates, such distinctions could have important practical implications (e.g., in personnel selection or vocational counselling). Similarly, within the Dark Triad, some researchers have argued for the existence of different types of psychopathy and narcissism (Porter et al., 2000; Wink, 1991), and there is some empirical evidence for this in the case of narcissism (Wetzel, Leckelt, Gerlack, \& Back, 2016).

The forgoing discussion illustrates only a few of the ways that a person-centered approach might be applied to complement and extend the large body of variable-centered personality research. As we noted at the outset, perhaps the biggest advantage of the person-centered approach is that it helps to identify differences in the ways that personality traits combine and might therefore be expressed. We see evidence of this in our own study. For example, previous research has shown that Agreeableness and Honesty-Humility are typically positively correlated (Ashton \& Lee, 2009; Lee \& Ashton, 2016). Although this relationship is expressed as expected in our Socially Considerate, Goal-Oriented, and Maladjusted profiles, the relationship is unexpectedly reversed in the context of the Self-confident profile 
wherein a high level of Agreeableness is accompanied by a low level of Honesty-Humility. Similarly, we see above average levels of Extraversion accompanied by above average Agreeableness in the Selfconfident profile, but with below average Agreeableness in the Maladjusted profile. The way these traits manifest themselves across profiles could therefore be quite different. Thus, there may be advantages to re-examining many of the previous finding from variable-centered research to determine whether the observed relations might be more nuanced than previously believed.

\subsection{Conclusions}

In conclusion, the results of our study provide additional evidence for the existence of multiple subgroups with different trait dimension profiles within a population. Although the profiles we identified differed from those observed by Isler et al. (2016) for the HEXACO, the evidence we provided for consistency across samples suggest that they are indeed meaningful within the population under investigation. We recognize that the profiles we found might represent only a subset of all possible profiles within the HEXACO framework. Accordingly, we call for a broader application of a personcentered approach to replicate and extend our findings. We also encourage further investigation of the psychological processes underlying the formation of the personality profiles and their implications for emotion, attitudes, and behavior.

\section{References}

Aghababaei, N., \& Arji, A. (2014). Well-being and the HEXACO model of personality. Personality and Individual Differences, 56, 139-142.

Akaïke, H. (1987). Factor analysis and AIC. Psychometrika, 52, 317-332.

Asendorpf, J.B. (2015). Person-centered approaches to personality. In M. Mikulincer, P.R. Shaver, L.M. Cooper, \& R.J. Larsen (Eds.), APA Handbook of Personality and Social Psychology Volume 4: Personality Processes and Individual Differences (pp. 403-424). Washington, DC: American Psychological Association.

Asendorpf, J. B., Borkenau, P., Ostendorf, F., \& Van Aken, M. A. (2001). Carving personality description at its joints: Confirmation of three replicable personality prototypes for both children and adults. European Journal of Personality, 15(3), 169-198.

Ashton, M. C., Jackson, D. N., Paunonen, S. V., Helmes, E., \& Rothstein, M. G. (1995). The criterion validity of broad factor scales versus specific facet scales. Journal of Research in Personality, 29(4), 432-442.

Ashton, M. C., \& Lee, K. (2007). Empirical, theoretical, and practical advantages of the HEXACO model of personality structure. Personality and Social Psychology Review, 11(2), 150-166.

Ashton, M. C., \& Lee, K. (2005). Honesty humility, the big five, and the five factor model. Journal of Personality, 73(5), 1321-1354.

Ashton, M. C., \& Lee, K. (2008). The prediction of honesty-humility-related criteria by the HEXACO and five-factor models of personality. Journal of Research in Personality, 42(5), 12161228.

Ashton, M. C., \& Lee, K. (2009). An investigation of personality types within the HEXACO personality framework. Journal of Individual Differences, 30(4), 181-187.

Barbaranelli, C. (2002). Evaluating cluster analysis solutions: An application to the Italian NEO personality inventory. European Journal of Personality, 16, 43-55.

Block, J. (1971). Lives through time. Berkeley, CA: Bancroft Books.

Block, J. H., \& Block, J. (1980). The role of ego-control and egoresiliency in the organization of behavior. In W. A. Collins (Eds.), Minnesota Symposium on Child Dsychology (pp. 39-101),13. Hillsdale, NJ: Erlbaum.

Bourdage, J.S., Lee, K., Lee, J.H., \& Shin, K.H. (2012). Motives for organizational citizenship behavior: Persoanlity correlates and coworker ratings of OCB. Human Performance, 25, 179-200.

Bozdogman, H. (1987). Model selection and akaike's information criterion (AIC): The general theory and its analytical extensions. Psychometrika, 52, 345-370.

Chapman, B. P., \& Goldberg, L. R. (2011). Replicability and 40-year predictive power of 
childhood ARC types. Journal of Personality and Social Psychology, 101(3), 593.

Conte, J. M., Heffner, T. S., Roesch, S. C., \& Aasen, B. (2017). A person-centric investigation of personality types, job performance, and attrition. Personality and Individual Differences, 104, 554-559.

De Vries, R. E. (2012). Personality predictors of leadership styles and the self-other agreement problem. The Leadership Quarterly, 23(5), 809-821.

De Vries, R.E., De Vries, A., De Hoogh, A., \& Feij, J. (2009). More than the Big Five: Egoism and the HEXACO model of personality. European Journal of Personality, 23, 635-654.

Donnellan, M. B., Oswald, F. L., Baird, B. M., \& Lucas, R. E. (2006). The mini-IPIP scales: Tiny-yeteffective measures of the Big Five factors of personality. Psychological Assessment, 18(2), 192.

Gramzow, R. H., Sedikides, C., Panter, A. T., Sathy, V., Harris, J., \& Insko, C. A. (2004). Patterns of self-regulation and the Big Five. European Journal of Personality, 18(5), 367-385.

Herzberg, P. Y., \& Roth, M. (2006). Beyond resilients, undercontrollers, and overcontrollers? An extension of personality prototype research. European Journal of Personality, 20(1), 5-28.

Isler, L., Liu, J. H., Sibley, C. G., \& Fletcher, G. J. (2016). Self-regulation and personality profiles: empirical development, longitudinal stability and predictive ability. European Journal of Personality, 30(3), 274-287.

Jackson, D. N., Paunonen, S. V., Fraboni, M., \& Goffin, R. D. (1996). A five-factor versus sixfactor model of personality structure. Personality and Individual Differences, 20(1), 33-45.

Kabins, A. H., Xu, X., Bergman, M. E., Berry, C. M., \& Willson, V. L. (2016). A profile of profiles: A meta-analysis of the nomological net of commitment profiles. Journal of Applied Psychology, 101(6), 881-904.

Johnson, M. K., Rowatt, W. C., \& Petrini, L. (2011). A new trait on the market: HonestyHumility as a unique predictor of job performance ratings. Personality and Individual Differences, 50(6), 857-862.

Jones, D. N., \& Paulhus, D. L. (2014). Introducing the short dark triad (SD3) a brief measure of dark personality traits. Assessment, 21(1), 28-41.

Lee, K., \& Ashton, M. C. (2004). Psychometric properties of the HEXACO personality inventory. Multivariate Behavioral Research, 39(2), 329-358.

Lee, K., \& Ashton, M. C. (2005). Psychopathy, machiavellianism, and narcissism in the fivefactor model and the HEXACO model of personality structure. Personality and Individual Differences, 38(7), 1571-1582.

Lee, K., \& Ashton, M. C. (2014). The dark triad, the big five, and the HEXACO model. Personality and Individual Differences, 67, 2-5.

Lee, K., \& Ashton, M. C. (2016). Psychometric Properties of the HEXACO-100. Assessment, 115.

Lee, K., Ashton, M.C., \& De Vries, R.E. (2005). Predicting workplace delinquency and integrity with the HEXACO and Five-Factor Models of personality structure. Human Performance, 18, 179-197.

Lo, Y., Mendell, N., \& Rubin, D. (2001). Testing the number of components in a normal mixture. Biometrika, 88, 767-778.

Lubke, G. H., \& Muthén, B. O. (2007). Performance of factor mixture models as a function of model size, covariate effects, and class-specific parameters. Structural Equation Modeling, 14(1), 26-47.

Marcus, B., Ashton, M. C., \& Lee, K. (2013). A Note on the Incremental Validity of Integrity Tests beyond Standard Personality Inventories for the Criterion of Counterproductive Behaviour. Canadian Journal of Administrative Sciences, 30, 18-25.

Marcus, B., Lee, K., \& Ashton, M. C. (2007). Personality dimensions explaining relationships between integrity tests and counterproductive behavior: Big five, or one in addition? Personnel Psychology, 60(1), 1-34.

Marsh, H. W., Lüdtke, O., Trautwein, U., \& Morin, A. J. S. (2009). Classical latent profile analysis of academic self-concept dimensions: Synergy of person- and variable-centered approaches to theoretical models of self-concept. Structural Equation Modeling, 16, 191-225. 
McClelland, G. H., \& Judd, C. M. (1993). Statistical difficulties of detecting interactions and moderator effects. Psychological Bulletin, 114(2), 376.

McLachlan, G., \& Peel, D. A. (2000). Finite mixture models. New York: Wiley.

Meade, A. W., \& Craig, S. B. (2012). Identifying careless responses in survey data. Psychological Methods, 17(3), 437.

Meyer, J.P., \& Morin, A.J.S. (2016). A person-centred approach to commitment research: Theory, research, and methodology. Journal of Organizational Behavior, 36, 584-612.

Merz, E. L., \& Roesch, S. C. (2011). A latent profile analysis of the five factor model of personality: Modeling trait interactions. Personality and Individual Differences, 51(8), 915-919.

Morin, A. J., Meyer, J. P., Creusier, J., \& Biétry, F. (2016). Multiple-group analysis of similarity in latent profile solutions. Organizational Research Methods, 19, 231-254.

Morin, A. J., Morizot, J., Boudrias, J. S., \& Madore, I. (2011). A multifoci person-centered perspective on workplace affective commitment: A latent profile/factor mixture analysis. Organizational Research Methods, 14(1), 58-90.

Muthén, B. O. (2001). Latent variable mixture modeling. In G. A. Marcoulides \& R. E. Schumacker (Eds.), New developments and techniques in structural equation modeling (pp. 1-33): Laurence Erlbaum Associates.

Muthén, L. K., \& Muthén, B. O. (1998-2012). Mplus User's Guide (Seventh ed.). Los Angeles, CA: Muthén \& Muthén.

Nylund, K. L., Asparouhov, T., \& Muthén, B. O. (2007). Deciding on the number of classes in latent class analysis and growth mixture modeling: A monte carlo simulation study. Structural Equation Modeling, 14, 535-569.

Pastor, D. A., Barron, K. E., Miller, B. J., \& Davis, S. L. (2007). A latent profile analysis of college students' achievement goal orientation. Contemporary Educational Psychology, 32, 8-47.

Paulhus, D. L., \& Williams, K. M. (2002). The dark triad of personality: Narcissism, machiavellianism, and psychopathy. Journal of Research in Personality, 36, 556-563.

Paunonen, S. V. (1998). Hierarchical organization of personality and prediction of behavior. Journal of Personality and Social Psychology, 74(2), 538.

Paunonen, S. V., \& Ashton, M. C. (2001). Big five factors and facets and the prediction of behavior. Journal of Personality and Social Psychology, 81(3), 524.

Paunonen, S. V., Rothstein, M. G., \& Jackson, D. N. (1999). Narrow reasoning about the use of broad personality measures for personnel selection. Journal of Organizational Behavior, 389405.

Porter, S., Fairweather, D., Drugge, J., Hervé, H., Birt, A., \& Boer, D. (2000). Profiles of Psychopathy in incarcerated sexual offenders. Criminal Justice and Behavior, 27, 216-233.

Robins, R. W., John, O. P., Caspi, A., Moffitt, T. E., \& Stouthamer-Loeber, M. (1996). Resilient, overcontrolled, and undercontrolled boys: Three replicable personality types. Journal of Personality and Social psychology,70(1), 157.

Roth, M., \& von Collani, G. (2007). A head-to-head comparison of big-five types and traits in the prediction of social attitudes: Further evidence for a five-cluster typology. Journal of Individual Differences, 28(3), 138-149.

Schwartz, G. (1978). Estimating the dimension of a model. The Annals of Statistics, 6, 461-464.

Sclove, S. L. (1987). Application of model-selection criteria to some problems with multivariate analysis. Psychometrika, 52(3), 333-343.

Sibley, C. G., Harding, J. F., Perry, R., Asbrock, F., \& Duckitt, J. (2010). Personality and prejudice: Extension to the HEXACO personality model. European Journal of Personality, 24(6), 515-534.

Specht, J., Luhmann, M., \& Geiser, C. (2014). On the consistency of personality types across adulthood: Latent profile analyses in two large-scale panel studies. Journal of Personality and Social Psychology, 107(3), 540.

Vandenberg, R. J., \& Lance, C. E. (2000). A review and synthesis of the measurement invariance 
literature: Suggestions, practices, and recommendations for organizational research. Organizational Research Methods, 3(1), 4-70.

Veselka, L., Schermer, J. A., \& Vernon, P. A. (2011). Beyond the Big Five: The dark triad and the supernumerary personality inventory. Twin Research and Human Genetics, 14(02), 158-168.

Wang, M., \& Hanges, P. J. (2011). Latent class procedures: Applications to organizational research. Organizational Research Methods, 14(1), 24-31.

Wetzel, E., Leckelt, M., Gerlach, T. M., \& Back, M. D. (2016). Distinguishing subgroups of narcissists with latent class analysis. European Journal of Personality, 30(4), 374-389.

Wink, P. (1991). Two faces of narcissism. Journal of Personality and Social Psychology, 61, 590-597.

Zyphur, M. J. (2009). When mindsets collide: Switching analytical mindsets to advance organizational science. Academy of Management Review, 34, 677-688. 
Table 1

Descriptive Statistics for Samples $1^{a}$ and $2^{b}$

\begin{tabular}{lcccccccccccc}
\hline Variable & $M_{1}$ & $(S D)_{1}$ & $M_{2}$ & $(S D)_{2}$ & Age & HH & Emot. & Extra. & Agree. & Consc. & Open \\
\hline Age & 18.28 & $(1.53)$ & 18.37 & $(1.71)$ & - & $.11^{* * *}$ & -.04 & -.05 & $.08^{*}$ & -.01 & $.12^{* * *}$ \\
HH & 3.16 & $(.67)$ & 3.17 & $(.65)$ & $.08^{*}$ & $(.75 \backslash .77)$ & $.13^{* * *}$ & $-.12^{* * *}$ & $.30^{* * *}$ & $.13^{* * *}$ & .05 & \\
Emot. & 3.42 & $(.69)$ & 3.39 & $(.66)$ & $-.15^{* * *}$ & $.14^{* * *}$ & $(.79 \backslash .78)$ & $-.11^{* *}$ & -.05 & $.10^{* *}$ & -.04 & .04 \\
Extra. & 3.35 & $(.64)$ & 3.36 & $(.63)$ & -.06 & $-.13^{* * *}$ & $-.13^{* * *}$ & $(.79 \backslash .80)$ & .02 & $.13^{* * *}$ & .03 & .03 \\
Agree. & 3.15 & $(.65)$ & 3.17 & $(.61)$ & $.12^{* * *}$ & $.34^{* * *}$ & -.02 & .02 & $(.78 \backslash .76)$ & .02 & .03 \\
Consc. & 3.66 & $(.60)$ & 3.61 & $(.60)$ & .00 & $.17^{* * *}$ & $.18^{* * *}$ & $.12^{* * *}$ & .04 & $(.76 \backslash .78)$ & -.02 \\
Open & 3.22 & $(.66)$ & 3.22 & $(.63)$ & $.17^{* * *}$ & .05 & $-.13^{* * *}$ & $.09^{*}$ & $.09^{* *}$ & -.02 & $(.74 \backslash .73)$
\end{tabular}

Note: ${ }^{a} n=880 ;{ }^{b} n=858$; Subscripts after $M$ and $S D$ indicate sample; ${ }^{*}$ denotes significance at $p<.05,{ }^{* *}$ at $p<.01$, and ${ }^{* * *}$ at $p<.001$. Correlations for Sample 1 are above the diagonal and those for Sample 2 are below the diagonal. Reliabilities are reported on the diagonal. $\mathrm{HH}=$ Honesty-Humility; Emot. = Emotionality; Extra. = Extraversion; Agree. = Agreeableness; Consc. $=$ Conscientiousness; Open $=$ Openness 
Table 2

Model Fit Statistics for the 2- to 8-Profile Models

\begin{tabular}{|c|c|c|c|c|c|c|c|c|c|}
\hline & LL & $\mathrm{SC}$ & $\mathrm{AIC}$ & $\mathrm{BIC}$ & CAIC & SABIC & Entropy & LMR & BLRT \\
\hline \multicolumn{10}{|l|}{ Sample 1} \\
\hline 2-profile & -6762.737 & 1.1566 & 13563.475 & 13654.293 & 13600.419 & 13593.954 & 0.516 & $\leq .001$ & $\leq .001$ \\
\hline 3-profile & -6721.186 & 1.2467 & 13494.373 & 13618.65 & 13544.928 & 13536.08 & 0.566 & 0.092 & $\leq .001$ \\
\hline 4-profile & -6685.847 & 1.1430 & 13437.694 & 13595.431 & 13501.861 & 13490.631 & 0.607 & 0.021 & $\leq .001$ \\
\hline 5-profile & -6652.577 & 1.2763 & 13385.153 & 13576.35 & 13462.933 & 13449.319 & 0.622 & 0.325 & $\leq .001$ \\
\hline 6-profile & -6633.492 & 1.3125 & 13360.984 & 13585.641 & 13452.374 & 13436.379 & 0.656 & 0.363 & $\leq .001$ \\
\hline 7-profile & -6616.895 & 1.3283 & 13341.789 & 13599.905 & 13446.792 & 13428.413 & 0.657 & 0.425 & $\leq .001$ \\
\hline 8-profile & -6599.715 & 1.4341 & 13321.43 & 13613.01 & 13440.000 & 13419.28 & 0.67 & 0.733 & $\leq .001$ \\
\hline \multicolumn{10}{|l|}{ Sample 2} \\
\hline 2-profile & -6385.211 & 1.1246 & 12808.422 & 12898.759 & 12845.367 & 12838.421 & 0.605 & $\leq .001$ & $\leq .001$ \\
\hline 3-profile & -6331.301 & 1.3913 & 12714.602 & 12838.222 & 12765.158 & 12755.653 & 0.641 & 0.217 & $\leq .001$ \\
\hline 4-profile & -6288.305 & 1.3035 & 12642.61 & 12799.512 & 12706.777 & 12694.713 & 0.656 & 0.122 & $\leq .001$ \\
\hline 5-profile & -6257.545 & 1.9557 & 12595.089 & 12785.274 & 12672.869 & 12658.245 & 0.669 & 0.871 & $\leq .001$ \\
\hline 6-profile & -6227.77 & 1.3079 & 12549.541 & 12773.007 & 12640.930 & 12623.748 & 0.654 & 0.041 & $\leq .001$ \\
\hline 7-profile & -6207.552 & 2.0334 & 12523.104 & 12779.852 & 12628.106 & 12608.363 & 0.662 & 0.937 & $\leq .001$ \\
\hline 8-profile & -6185.299 & 1.2592 & 12492.597 & 12782.628 & 12611.200 & 12588.909 & 0.708 & 0.171 & $\leq .001$ \\
\hline
\end{tabular}

Note. $\mathrm{LL}=$ Model loglikelihood; $\mathrm{SC}=$ Scaling correction factor; $\mathrm{AIC}=$ Akaïe information criterion; CAIC $=$

Consistent AIC; BIC = Bayesian information criterion; SABIC $=$ Sample-size adjusted BIC; LMR $=$ Lo-MendellRubin likelihood ratio test; BLRT = Bootstrap likelihood ratio test. 
Table 3

Profile Membership of the 2- to 8-Profile Models

\begin{tabular}{lllllllll}
\hline \multicolumn{1}{c}{} & $\mathbf{1}$ & $\mathbf{2}$ & $\mathbf{3}$ & $\mathbf{4}$ & $\mathbf{5}$ & $\mathbf{6}$ & $\mathbf{7}$ & $\mathbf{8}$ \\
\hline Sample 1 & & & & & & & & \\
2-Profile & $41.90 \%$ & $58.10 \%$ & & & & & & \\
3-Profile & $21.56 \%$ & $28.14 \%$ & $50.31 \%$ & & & & & \\
4-Profile & $33.56 \%$ & $22.15 \%$ & $9.74 \%$ & $34.55 \%$ & & & & \\
5-Profile & $9.40 \%$ & $22.80 \%$ & $33.43 \%$ & $7.26 \%$ & $27.10 \%$ & & & \\
6-Profile & $8.92 \%$ & $21.63 \%$ & $27.65 \%$ & $6.351 \%$ & $2.90 \%$ & $32.37 \%$ & & \\
7-Profile & $12.17 \%$ & $3.35 \%$ & $20.93 \%$ & $6.95 \%$ & $31.55 \%$ & $22.11 \%$ & $2.94 \%$ & \\
8-Profile & $4.14 \%$ & $19.64 \%$ & $28.46 \%$ & $7.96 \%$ & $11.57 \%$ & $1.11 \%$ & $24.10 \%$ & $3.04 \%$ \\
\hline Sample 2 & & & & & & & & \\
2-Profile & $39.30 \%$ & $60.70 \%$ & & & & & & \\
3-Profile & $13.56 \%$ & $31.63 \%$ & $54.81 \%$ & & & & & \\
4-Profile & $41.82 \%$ & $32.52 \%$ & $7.69 \%$ & $18.97 \%$ & & & & \\
5-Profile & $6.00 \%$ & $7.94 \%$ & $30.57 \%$ & $14.16 \%$ & $41.33 \%$ & & & \\
6-Profile & $27.91 \%$ & $7.86 \%$ & $11.76 \%$ & $3.21 \%$ & $32.50 \%$ & $16.77 \%$ & & \\
7-Profile & $4.25 \%$ & $30.00 \%$ & $25.39 \%$ & $20.19 \%$ & $6.87 \%$ & $10.80 \%$ & $2.5 \%$ & \\
8-Profile & $2.65 \%$ & $17.23 \%$ & $11.36 \%$ & $4.91 \%$ & $35.99 \%$ & $5.84 \%$ & $6.12 \%$ & $15.90 \%$ \\
\hline
\end{tabular}


Table 4

Classification Posterior Probabilities for the 5-Profile Model

\begin{tabular}{lccccc}
\hline Profile & Profile 1 & Profile 2 & Profile 3 & Profile 4 & Profile 5 \\
\hline Sample 1 & & & & & \\
1 & .71 & .07 & .09 & .02 & .13 \\
2 & .02 & .74 & .07 & .04 & .13 \\
3 & .03 & .07 & .81 & .00 & .09 \\
4 & .00 & .11 & .00 & .79 & .09 \\
5 & .06 & .10 & .09 & .02 & .72 \\
\hline Sample 2 & & & & & .00 \\
1 & .85 & .01 & .09 & .05 & .05 \\
2 & .01 & .76 & .16 & .03 & .10 \\
3 & .03 & .07 & .73 & .07 & .08 \\
4 & .03 & .03 & .13 & .73 & .84 \\
5 & .00 & .02 & .10 & .04 & \\
\hline
\end{tabular}

Note: Values in bold are the average posterior probabilities associated with the profiles to which individuals were assigned. 
Table 5

Invariance Test Statistics for the 5-Profile Model

\begin{tabular}{lccccccc}
\hline & LL & SC & AIC & BIC & CAIC & SABIC & Entropy \\
\hline Configural & -14114.672 & 1.6084 & 28391.344 & 28833.643 & 28572.788 & 28576.314 & 0.752 \\
Structural & -14128.541 & 1.2985 & 28359.082 & 28637.567 & 28473.324 & 28475.545 & 0.738 \\
Dispersion & -14138.829 & 1.3426 & 28367.658 & 28613.38 & 28468.460 & 28470.419 & 0.74 \\
Distributional & -14139.875 & 1.3432 & 28361.75 & 28585.63 & 28453.592 & 28455.377 & 0.739 \\
\hline
\end{tabular}

Note. $\mathrm{LL}=$ Model loglikelihood; $\mathrm{SC}=$ Scaling correction factor; $\mathrm{AIC}=$ Akaïke information criterion; $\mathrm{CAIC}=$ Consistent AIC; BIC = Bayesian information criterion; SABIC $=$ Sample-size adjusted BIC. 


\section{Five Profile Solution (Factor Scores)}

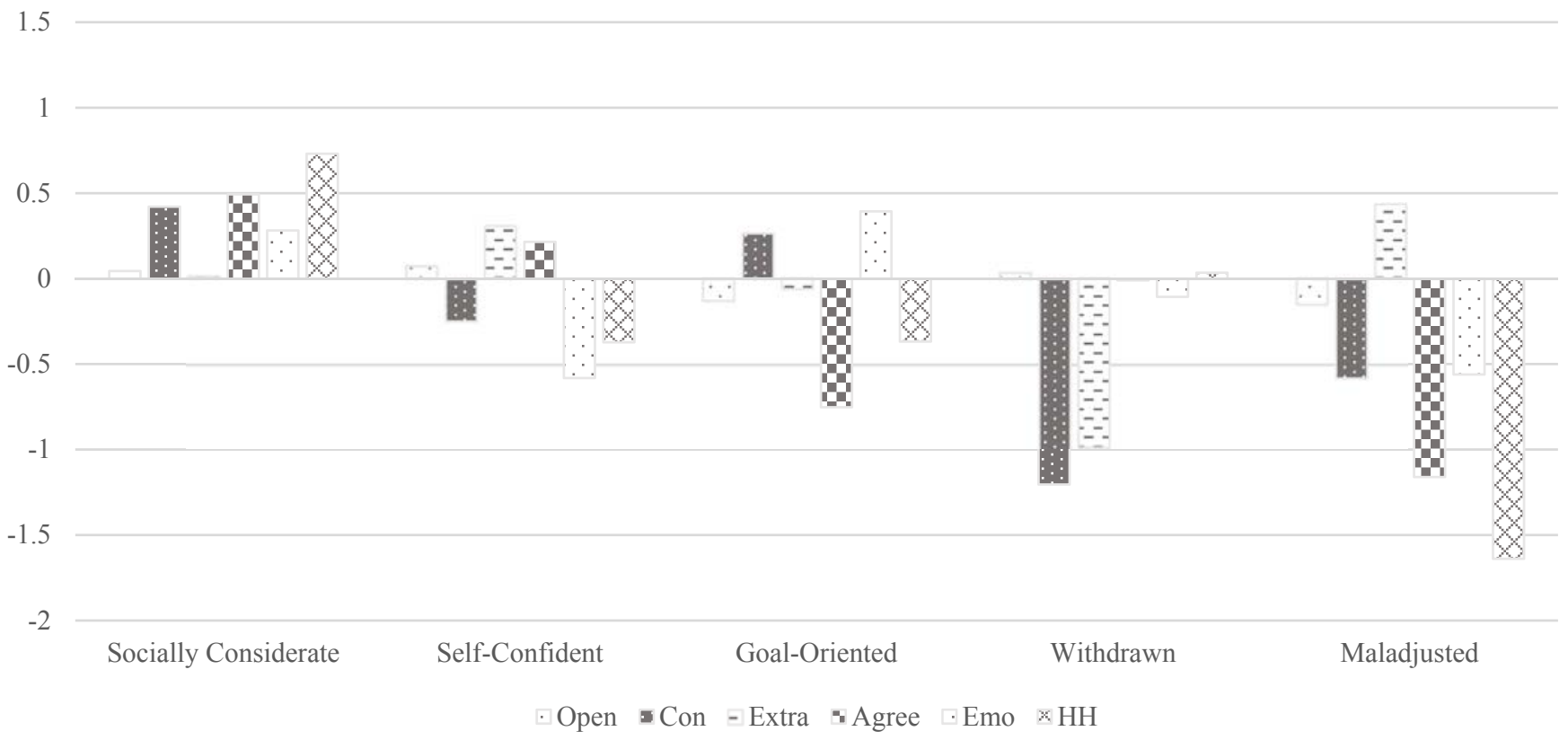

Figure 1. Means of the six HEXACO subscales for the 5-profile solutions expressed as factor scores. Samples 1 and 2. 